\title{
Non-Stem Amino Acids Are Involved in the Phage P22 TSP NTD Stability
}

\author{
Karthikeya Venkatesan, Jeremie Williams, Robert Villafane* \\ Department of Biological Sciences and Program in Microbiology, Alabama State University, Montgomery, \\ Alabama, USA \\ Email: ${ }^{*}$ villafane@alasu.edu
}

Received 5 May 2014; revised 7 June 2014; accepted 3 July 2014

Copyright @ 2014 by authors and Scientific Research Publishing Inc.

This work is licensed under the Creative Commons Attribution International License (CC BY). http://creativecommons.org/licenses/by/4.0/

(c) (i) Open Access

\section{Abstract}

The P22 phage system is an intensely studied model system. Studies have ranged from biochemical analysis of basic life processes to the use of this phage for phage therapy. The phage tailspike protein (TSP) has itself been the subject of intensive studies over the past fifty years. The P22 TSP is essential for initiation of the infection process and instrumental as the last protein assembled onto the phage particle structure to complete its assembly. It has also been the subject for many structural studies including cryoelectron microscopic analysis and photophysical studies. It has been a model for in vivo and in vitro protein folding including analysis using P22 TSP temperaturesensitive for folding mutations ( $t s f)$. Recently the structure and function of the $\mathrm{N}$-terminal domain (NTD), including some aspects of the structural stability of the P22 TSP NTD (aa1-aa108), are being genetically dissected. This report strongly supports the notion that two amino acids, not localized to the internal NTD dome stem, are important in the structural stability of the P22 TSP NTD.

\section{Keywords}

N-Terminal Domain (NTD), P22 Phage, Tailspike Protein (TSP), Mutagenesis, Structural Stability

\section{Introduction}

The bacteriophage (phage) P22 is a well-studied phage system as well as being an intensely used model for a number of life processes and proteins. P22 infects a very small number of Salmonella species, including Salmonella typhimurium. Its assembly pathway has been studied in great detail [1]-[3]. Cryoelectron microscopy has been used in recent years to describe the protein structure of phage particles in near atomic detail [4] [5]. The P22 phage is a member of the Podoviridae family of bacteriophages, having a short non-contractile phage tail

"Corresponding author. 
structure [6].

The P22 tailspike protein (TSP) is one of the most well-studied phage proteins. It is the last protein to be added in the phage assembly pathway to yield a completed infectious particle [7] [8]. This last assembly step, when done in vitro, is called tailing. The P22 TSP, the subject of this study, is a trimeric protein consisting of 666aa in which the first stable form of the tail protein is its trimeric form [9] [10]. In addition to its pivotal assembly role, the P22 TSP is responsible for selection and attachment of the phage to the bacterial host cell lipopolysaccharide (LPS) receptor [11].

The P22 TSP has several novel characteristics: resistance to sodium dodecyl sulfate (SDS) denaturation, resistant to heat $\left(\operatorname{Tm}\right.$ of $\left.88.3^{\circ} \mathrm{C}\right)$ and resistance to proteolysis [12] [13]. Each chain consists of three domains: the N-terminal domain (NTD, aa1-aa108), the $\beta$-helix (aa143-aa540) and the trimerization domain (aa541-aa666; see Figure 1). The P22 TSP NTD binds to the crevice formed between the GP4 and GP10 viral structural proteins while a crevice formed by two sets of peptide ridges, located in the $\beta$-helix domain is responsible for host cell LPS binding and hydrolysis [14] [15]. Recent studies on the structure and function of the P22 TSP NTD have found amino acids, located within the interior of the NTD dome, are involved in the stability of the domain structure [16]. This Short Communication presents preliminary studies strongly suggesting two amino acids, which are not localized in the stem region, and may be important determinants in the overall stability of the P22 TSP NTD.

\section{Materials and Methods}

\subsection{Primers and Mutagenesis}

The two tail mutations, studied in this Short Communication, were isolated by the Berget Laboratory [17]. They were studied here because they were the only two mutations found to be located in the P22 TSP NTD [17]. The P22 TSP NTD domain is an important research focus of this laboratory. They were: hmH4-1 (G29R; GGG < codon 29 > AGG) and hmH3 (G71D; GGT < codon $71>$ GAT). The following primers were used to transfer the Berget NTD mutations onto pET11a-TSP. The primers for the mutation hmH4-1 were: 5-G28R (or: aaagctgttgctaataggaaaatttacattggt) and 3-G28R (or: ac caatgtaaatttcctattagcaacagctt). The primers for the muta-

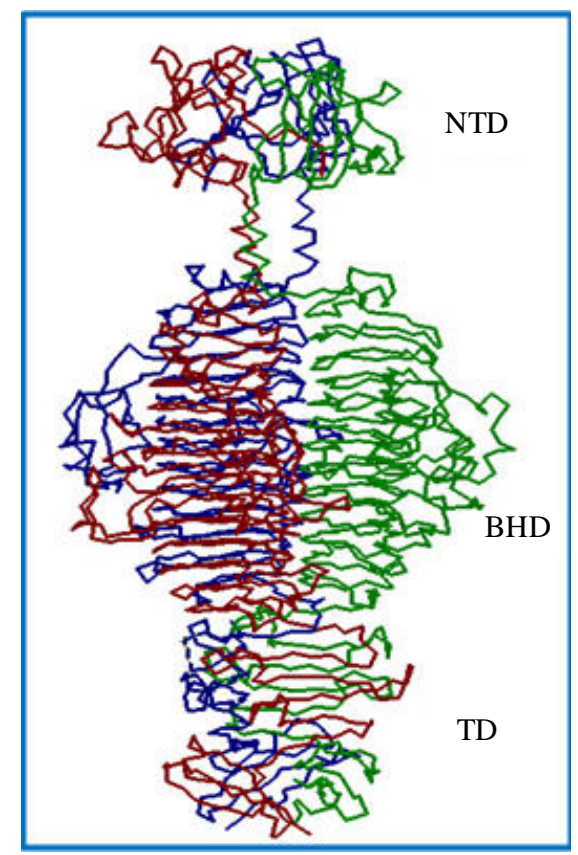

Figure 1. Trimeric structure of the P22 tailspike protein. The three domains of the P22 TSP are: the N-terminal domain (NTD), the beta-helix domain (BHD) and the trimerization domain (TD). 
tion hmH3 were: 5G70D (or: attatcaacgcagccgataaaatcgtatacaac) and 3G70D (or: gttgtatacgattttatcggctgcgttgataat). The tail mutations were transferred from primers to the pET11a-TSP DNA by the StratagenQuikChange Mutagenesis protocol. The E. coli strain BL21/DE3, used for gene expression, was from Novagen.

\subsection{Biological and Recombinant Protocols}

The biological, recombinant and expression protocols have been described [16] [18]. The expression plasmid, pET11a-TSP containing a wild type copy of the P22 tailspike gene, was from Dr Peter Prevelige (University of Alabama at Birmingham, UAB). The protein function of the expressed cloned tail gene or gene variant was determined by the Berget Assay [16]. The Berget Assay is a high-expression variant of the functional petri plate assay devised by Schwarz and Berget [17]. It has been described in detail recently [16].

\section{Results and Discussion}

\subsection{Mutations in the DNA Sequence Corresponding to the N-Terminal Domain}

Two previously identified mutations were localized to the gene region corresponding to the NTD. These mutations $h m H 4-1$ and $h m H 3$ were transferred to pET11a-TSP by the QuikChange Mutagenesis protocol and plasmid mutational sequences were confirmed by DNA sequencing using the sequencing facilities of the University of Alabama at Birmingham (UAB Heflin Genome Sequencing Facility).

\subsection{Assay of Cloned Tail Protein Function}

The functional nature of hmH4-1 (pET11a-TSP-G29R), hmH3 (pET11a-TSP-G71D) and the wild type tail gene (pET11a-TSP) was determined by the Berget Assay [16]. This test measures but does not distinguish between all three tail activities: binding to the viral particle (NTD function), binding LPS and LPS hydrolysis by tail endorhamnosidase activity (both functions of the $\beta$-helix domain). By this simple yet extremely sensitive petri plate assay these tail variants had no detectable tail function while the wild type tail gene, carried on plasmid, pET11a-TSP, had tail activity. These data confirmed the previous determination of the tail function of these two tail mutations [17]. The amino acid substitutions at amino acid positions aa29 and aa71 (out of 666aa) were both localized to the surface of the NTD [15]. To determine if the mutational inactivation of tail function could be due to structural alteration, a SDS-PAGE analysis was carried out as described in the following section.

\subsection{SDS PAGE Analysis}

Protein separation by SDS-PAGE normally results in denaturation of the applied proteins and their separation according to size. If the protein sample is heated prior to application to SDS PAGE, all protein will migrate according to their molecular size with the fastest migration occurring with the smaller proteins. However, there are a very small number of proteins that are refractory to SDS denaturation (without prior heating), including the P22 TSP [19]. The P22 TSP migrates as a trimeric protein with no prior heating [13]. However, there is another example of altered tailspike electrophoretic migration. Tailspike mutants have been found that destabilize the NTD and a thermal intermediate has been identified both of which destabilize the NTD resulting in the same migration which is slightly faster than the unheated trimeric TSP [16] [20] [21].

Thus the fully denatured, the NTD-denatured and wild type TSP conformations can be distinguished via electrophoretic migration study in SDS-PAGE with unheated samples. Such a study has been carried out and is depicted in Figure 2 and Figure 3. Figure 2 displays the partially purified extracts of WT TSP and TSP-G29K mutant protein while the gel figure (Figure 3 ) displays the partially purified extracts of WT TSP and TSP-G71D mutant protein. In both gels the WT TSP migrates above the $180 \mathrm{kDa}$ marker (TSP is $210 \mathrm{kDa}$ ). In both cases, the fully denatured TSP migrates below the $90 \mathrm{kDa}$ marker (TSP is $72 \mathrm{kDa}$ monomer). The unheated forms of the TSP-G29K and TSP-G71D (Figure 2, Lane 5 and Figure 3, Lane 4 respectively) migrate below the WT TSP but well above their denatured forms (Figure 2, Lane 3 and Figure 3, Lane 2 respectively). This is the location of the previously identified TSP forms whose NTD was denatured but not the other domains.

\section{Discussion}

The two sites G29K and G71D caused the N-terminal domain of the P22 TSP to collapse. Future studies will in- 


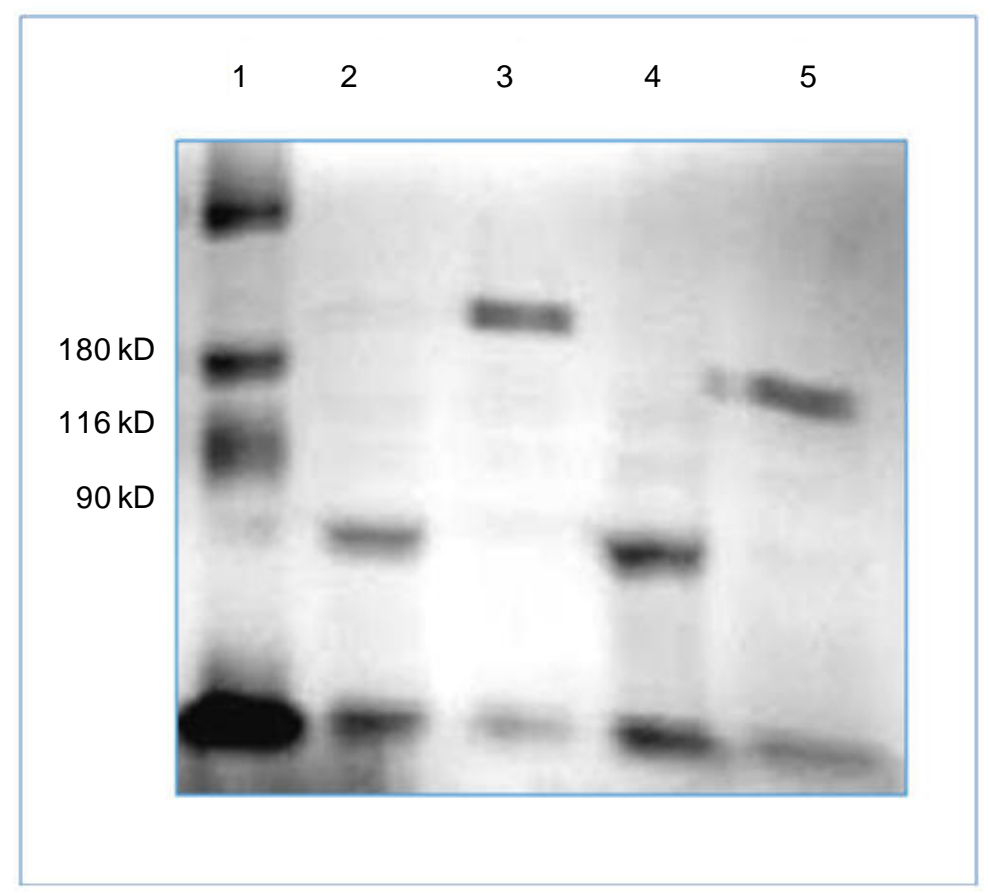

Figure 2. SDS-PAGE analysis of TSP-G29R. The gel contains Broad Range MW markers (Lane 1, L1), WT TSP (L2, L3), and TSP-G29R, (L4, L5) both heated and unheated.

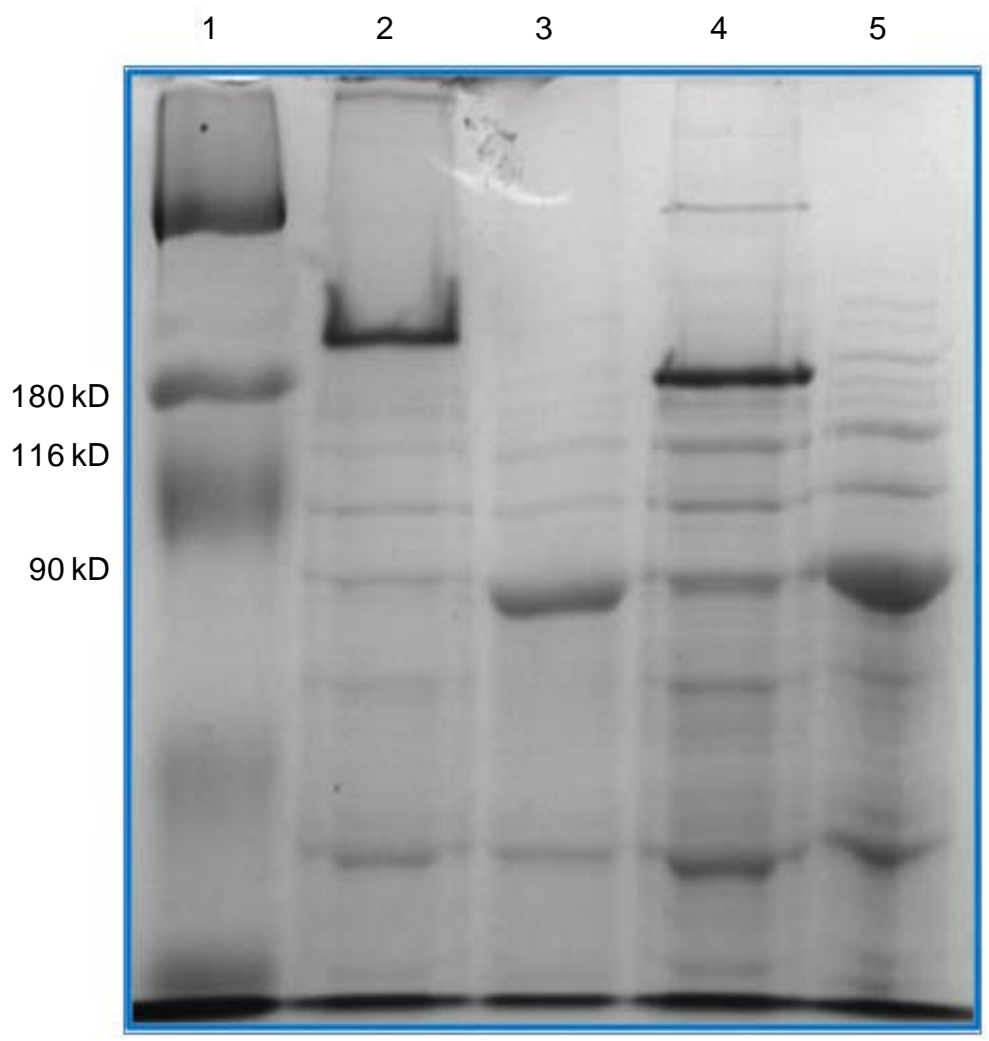

Figure 3. SDS-PAGE analysis of TSP-G71D. The gel contains Broad Range MW markers (Lane 1, L1), WT TSP (L2, L3), and TSP-G71D, (L4, L5) both unheated and heated. 
clude the determination of the nature of the two sites. It will be determined if these sites are tolerant to amino acid substitutions and the nature of the tolerated substitutions. Suppressor analyses will be carried out to determine if these two sites interact with other sites (intragenic suppressor analyses) in order to stabilize the P22 NTD.

\section{Conclusion}

This study strongly suggests that the amino acids at positions aa29 and aa71 are critical for the stability of the trimeric dome-like structure of the $\mathrm{N}$-terminal domain. Further studies are in progress.

\section{Acknowledgements}

The authors thank Drs Doba Jackson (Huntingdon College), B. K. Robertson (Alabama State University) and H. Wu (Alabama State University) for discussion. This study is partially supported by funds from Department of Biological Sciences of the College of Science, Mathematics and Technology and the Program in Microbiology of the Alabama State University.

\section{References}

[1] Aksyuk, A.A. and Rossmann, M.G. (2011) Bacteriophage Assembly. Viruses, 3, 172-203. http://dx.doi.org/10.3390/v3030172

[2] Casjens, S.R. and Thuman-Commike, P.A. (2011) Evolution of Mosaically Related Tailed Bacteriophage Genomes Seen through the Lens of Phage P22 Virion Assembly. Virology, 411, 393-415. http://dx.doi.org/10.3390/v3030172

[3] Strauss, H. and King, J. (1984) Steps in the Stabilization of Newly Packaged DNA during Phage P22 Morphogenesis. Journal of Molecular Biology, 172, 523-543. http://dx.doi.org/10.1016/S0022-2836(84)80021-2

[4] Hyrc, C.F., Chen, D.-H. and Chiu, W. (2011) Near-Atomic Resolution Cryo-EM for Molecular Virology. Current Opinion in Virology, 21, 110-117.

[5] Lander, G.C., Khayat, R., Li, R., Prevelige, P.E., Porter, C.S. and Carragher, B. (2009) The P22 Tail Machine at Subnanometer Resolution Reveals the Architecture of an Infectious Conduit. Structure, 17, 789-799. http://dx.doi.org/10.1016/j.str.2009.04.006

[6] Ackermann, H. (2009) Phage Classification and Characterization. Methods in Molecular Biology, 501, 127-140. http://dx.doi.org/10.1007/978-1-60327-164-6_13

[7] Israel, V., Anderson, T.F. and Levine, M. (1967) In Vitro Morphogenesis of Phage P22 from Heads and Base-Plate Parts. Proceedings of the National Academy of Sciences, 57, 284-291. http://dx.doi.org/10.1073/pnas.57.2.284

[8] Israel, V. (1978) Role of the Bacteriophage P22 Tail in the Early Stages of Infection. Journal of Virology, 18, $361-364$.

[9] Goldenberg, D.P. and King, J. (1982) Trimeric Intermediate in the in Vivo Folding and Subunit Assembly of the Tailspike Endorhamnosidase of Bacteriophage P22. Proceedings of the National Academy of Sciences, 79, 3403-3407. http://dx.doi.org/10.1073/pnas.79.11.3403

[10] Sauer, R.T., Krovatin, W., Poteete, A.R. and Berget, P.B. (1982) Phage P22 Tail Protein: Gene and Amino Acid Sequence. Biochemistry, 21, 5811-5815. http://dx.doi.org/10.1021/bi00266a014

[11] Shin, H., Lee, J.-H., Kim, H., Choi, Y., Heu, S. and Ryu, S. (2012) Receptor Diversity and Host Interaction of Bacteriophages Infecting Salmonella enteric SerovarTyphimurium. PLoS ONE, 7, e43392. http://dx.doi.org/10.1371/journal.pone.0043392

[12] Sturtevant, J.M., Yu, M.-H., Haase-Pettingell, C. and King, J. (1989) Thermostability of Temperature-Sensitive Folding Mutants of the P22 Tailspike Protein. The Journal of Biological Chemistry, 264, 10693-10698.

[13] Goldenberg, D.P., Berget, P.B. and King, J. (1982) Maturation of the Tail Spike Endorhamnosidase of Salmonella Phage P22. The Journal of Biological Chemistry, 257, 7864-7871.

[14] Andres, D., Hanke, C., Baxa, U., Seul, A., Barbirz, S. and Seckler, R. (2010) Tailspike Interactions with Lipopolysaccharide Effect: DNA Ejection from the Phage P22 Particles in Vitro. Journal of Biological Chemistry, 285, 3676836775. http://dx.doi.org/10.1074/jbc.M110.169003

[15] Steinbacher, S., Miller, S., Baxa, U., Budisa, N., Weintraub, A., Seckler, R. and Huber, R. (1997) Phage P22 Tailspike Protein: Crystal Structure of the Head-Binding Domain at $2.3 \AA$, Fully Refined Structure of the Endorhamnosidase at $1.56 \AA$ Resolution, and the Molecular Basis of O-Antigen Recognition and Cleavage. Journal of Molecular Biology, 267, 865-880. http://dx.doi.org/10.1006/jmbi.1997.0922

[16] Palmer, C., Williams, J., Dean, D., Johnson, S., Wu, H., Robertson, B.K., Jackson, D. and Villafane, R. (2014) Stem 
Mutants in the N-Terminal Domain of the Phage P22 Tailspike Protein. American Journal of Microbiological Research, 2, 1-7. http://dx.doi.org/10.12691/ajmr-2-1-1

[17] Schwarz, J.J. and Berget, P. (1989) The Isolation and Sequence of Missense and Nonsense Mutations in the Cloned Bacteriophage P22 Tailspike Protein Gene. Genetics, 121, 635-649.

[18] Sambrook, J., Fritsch, E. and Maniatis, T. (1989) Molecular Cloning. 2nd Edition, Cold Spring Harbor Laboratory Press, Plainview.

[19] Manning, M. and Colon, W. (2004) Structural Basis of Protein Kinetic Stability: Resistance to Sodium Dodecyl Sulfate Suggests a Central Role for Rigidity and a Bias Toward-Sheet Structure. Biochemistry, 43, 11248-1125. http://dx.doi.org/10.1021/bi0491898

[20] Maurides, P.A., Schwarz, J.J. and Berget, P.B. (1990) Intragenic Suppression of a Capsid Assembly-Defective P22 Tailspike Mutation. Genetics, 125, 673-681.

[21] Chen, B.-L. and King, J. (1991) Thermal Unfolding Pathway for the Thermostable P22 Tailspike Endorhamnosidase. Biochemistry, 30, 6260-6269. http://dx.doi.org/10.1021/bi00239a026 
Scientific Research Publishing (SCIRP) is one of the largest Open Access journal publishers. It is currently publishing more than 200 open access, online, peer-reviewed journals covering a wide range of academic disciplines. SCIRP serves the worldwide academic communities and contributes to the progress and application of science with its publication.

Other selected journals from SCIRP are listed as below. Submit your manuscript to us via either submit@scirp.org or Online Submission Portal.
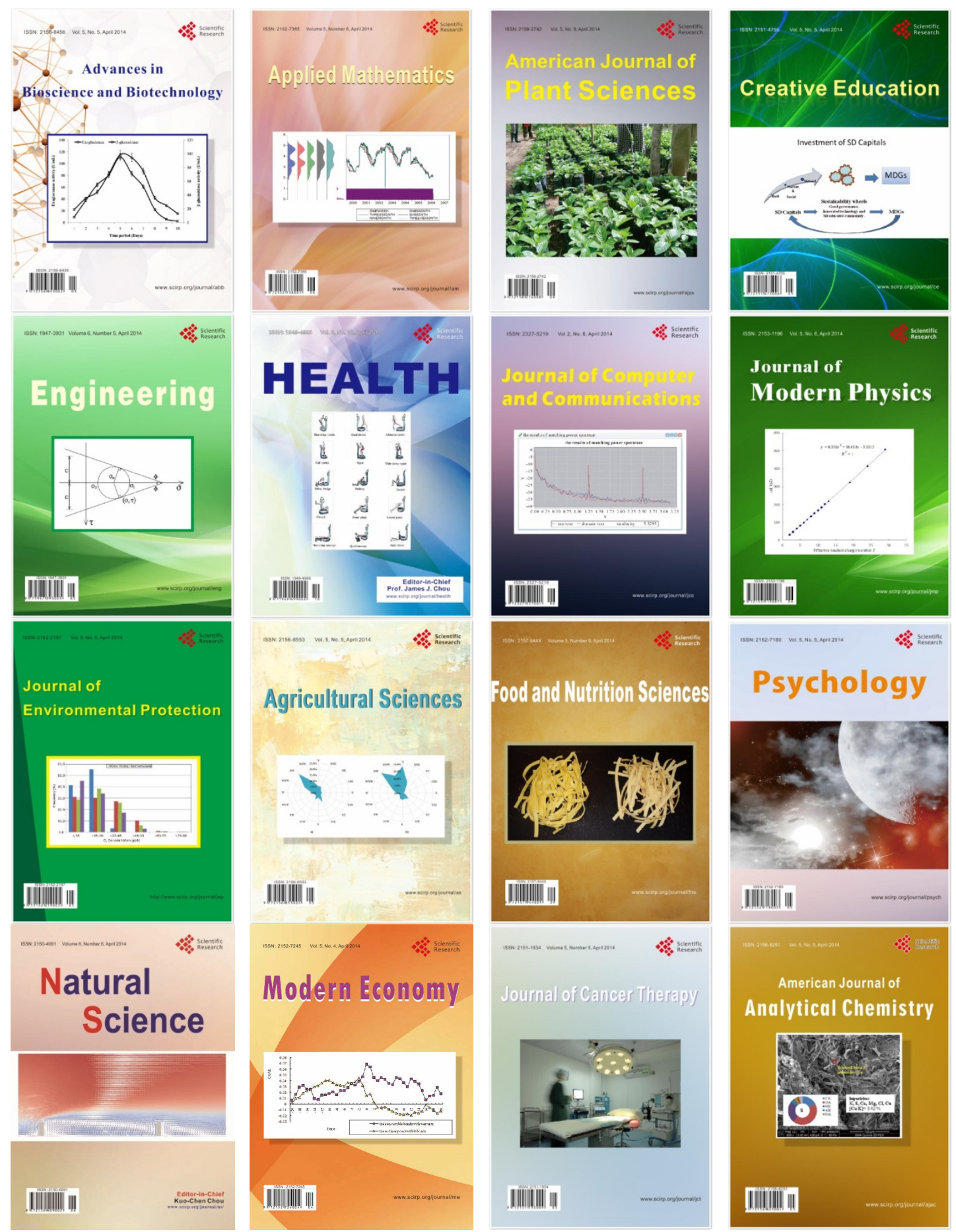